Int. J. Electrochem. Sci., 15 (2020) $9532-9542$

\title{
Characteristic and Control of Electrochemical Oscillation at the Anode during Electrolytic Refining Copper
}

\author{
Lifeng Ding ${ }^{=1,3, *}$, Qiang $\mathrm{Li}^{=, 2, *}$, Jinxia Yuan ${ }^{3}$, Xiaoyun Dong ${ }^{1}$, Di Peng ${ }^{4}$, Baoyi $\mathrm{Li}^{1}$, \\ Hongdao Li ${ }^{1}$, Yanfeng Xue ${ }^{1}$, Yulan Niu ${ }^{l}$ \\ ${ }^{1}$ Department of Chemistry and Chemical Engineering, Taiyuan Institute of Technology, Taiyuan \\ 030008, PR China \\ ${ }^{2}$ MicroNano System Research Center, College of Information and Computer \& Key Laboratory of Ad \\ vanced Transducers and Intelligent Control System of Ministry of Education and Shanxi Province, Tai \\ yuan University of Technology, Taiyuan, 030024, PR China \\ ${ }^{3}$ School of Chemical Engineering and Technology, North University of China, Taiyuan 030051, PR \\ China \\ ${ }^{4}$ Criminal Investigation College, Southwest University of Political Science and Law, Chongqing \\ 401120, PR China \\ =These authors contributed equally to this work. \\ *E-mail: happydlf@163.com, liqiang02@tyut.edu.cn.
}

doi: $10.20964 / 2020.09 .85$

Received: 4 June 2020 / Accepted: 20 July 2020 / Published: 10 August 2020

Electrolytic refining copper production is a high-energy hydrometallurgical industry. The key to develop a new method for energy-saving electrolysis of high-purity cathode copper is to reduce the electricalenergy consumption of electrochemical oscillations (EOs) at the anode. This phenomenon cannot be explained based on traditional equilibrium thermodynamics theory. In this paper, a simplified electrolytic copper system was used with $\mathrm{H}_{2} \mathrm{SO}_{4}-\mathrm{CuSO}_{4}$ solution to research the behaviour and characteristic of EOs at the anode, as well as to analyse the mechanism of EOs and the effect of potential on EOs.The electrochemical oscillation was regulated by improving the anode (such as copper anode and lead dioxide anode) and introducing additives (such as hydrochloric acid, thiourea and bone glue). The obvious inhibition effect of thiourea and lead dioxide anode on electrochemical oscillation was found, so as to improve the electrolysis process. It is possible to find a new energy-saving and environmental protection electrolysis working area in the equilibrium zone.

Keywords: Electrochemical oscillation, Electrolytic refining, Copper anode, Electrodissolution, Energy-saving

FULL TEXT 
(C) 2020 The Authors. Published by ESG (www.electrochemsci.org). This article is an open access article distributed under the terms and conditions of the Creative Commons Attribution license (http://creativecommons.org/licenses/by/4.0/). 